Echocardiographic trace of a racing cyclist.

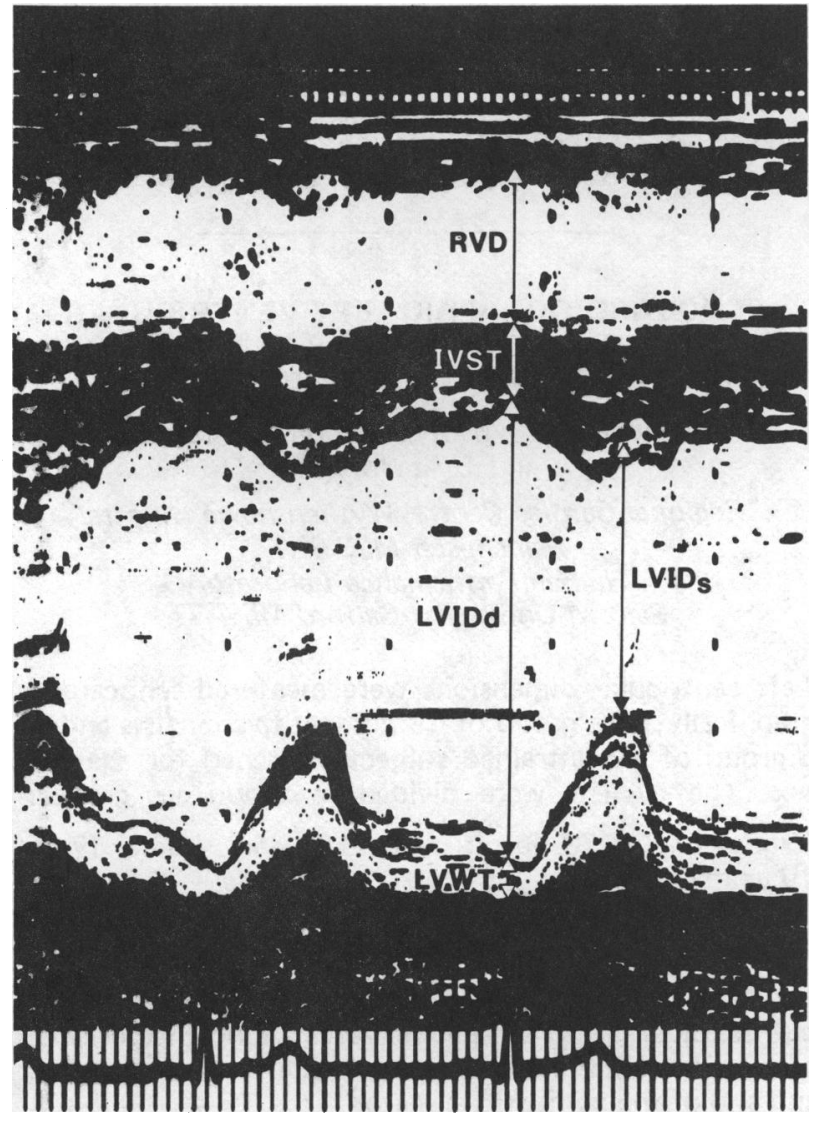

RVD Right Ventricular Dimension

IVST Inter Ventricular Septal Thickness

LVIDd Left Ventricular Internal Dimension (diastole)

LVIDs Left Ventricular Internal Dimension (systole)

LVWT Left Ventricular Wall Thickness

mass by 82 per cent and their left ventricular volumes by 20 per cent.

The cardiac adaptive changes to exercise were significantly different ( $p<0.02$ ) in the two age groups of cyclists. The older age group had larger left ventricular volumes and mass at the start of the season, but the changes in these parameters, as a result of training were more striking in the younger age group, by the end of the season (Table)

\section{PHYSIOLOGICAL AND SLEEP PATTERN RESPONSES TO A SUCCESSFUL ATTEMPT ON THE WORLD RECORD FOR CONTINUOUS WALKING}

\author{
Ann Daggett*, B. Davies*, C. Shapiro** and P. Jakemant \\ *Metabolic Profile Laboratory, University of Salford \\ * "Dept. of Psychiatry, Royal Edinburgh Hospital \\ tDept. of Sports Studies, \\ Crewe and Alsager College of Higher Education
}

Physiological responses to 130 hours of non-stop walking and sleep deprivation were recorded during and following a successful attempt on the world continuous walking record by an experienced and highly trained male walker (aged 47 years).

Heart rate, monitored continuously throughout the marathon walk, averaged $160 \mathrm{~b} / \mathrm{min}$ over the 6 days and 5 nights. The subject walked at approximately $55 \%$ of maximum oxygen uptake $\left(\mathrm{VO}_{2}\right.$ max), with lactate levels remaining below $2 \mathrm{mmol} / \mathrm{l}$. The total distance walked was 338.2 miles at a mean rate of $\mathbf{3 . 5} \mathrm{mph}$ during the first 24 hours, declining to $2 \mathrm{mph}$ over the final 24 hours.

Creatine kinase (CK) efflux reflected the changes in walking intensity, and the ratio of creatine kinase $M B$ to total CK (CK-MB/CK\%) indicated no evidence of cardiac ischaemia. Urine catecholamine levels rose throughout the walk period, with adrenaline, noradrenaline and dopamine increasing 9, 7 and 10 fold, respectively.

Adrenaline remained high during the 3 day recovery phase, whereas nor-adrenaline and dopamine had returned to their resting levels after 20 hours. Serum electrolyte changes were within normal ranges, and growth hormone $(\mathrm{GH})$ and prolactin (PRL) were raised but variable throughout the walk phase. Haemoglobin (Hb) and pack cell volume (PCV) levels decreased from $13.0-11.6 \mathrm{~g} / \mathrm{dl}$ and $42-34 \%$ respectively.

Following the walk, the subject demonstrated very short sleep latency, rapid entry into slow wave sleep (SWS) and rises in GH. The relative and absolute demands of SWS remained high for 3 nights. Total GH on the first post-walk day was increased $93 \%$ above baseline levels recorded during sleep, when the subject was not training. The extreme increase in SWS and rise in $\mathrm{GH}$ supports the sleep restorative hypothesis of $\mathrm{GH}$ release post-exercise and following sleep deprivation.

It would seem that a walk of this intensity is well within the physiological capabilities of an individual's aerobic capacity. However, the enormous psychological factor in overcoming sleep-deprivation is clearly illustrated in the post-exercise sleep patterns. 